\title{
Trapianto pecora-uomo: non è solo questione di bioetica
}

Giornale di Tecniche Nefrologiche e Dialitiche 2018, Vol. 30(2) 145-146

(C) The Author(s) 2018

Article reuse guidelines:

sagepub.com/journals-permissions

DOI: $10.1177 / 0394936218766853$

journals.sagepub.com/home/gtn

(3SAGE

\section{Mario Timio}

Sembrava una delle tante fake news che invadono minuto dopo minuto la rete. Non è così; questa volta sembra realtà. Un team della Università di Stanford, degli Stati Uniti d'America, è riuscito a far crescere per tre settimane embrioni che contengono cellule di uomo e cellule di pe-cora. È il primo passo per sviluppare in animali un numero illimitato di organi da trapiantare nell'uomo - a partire dal pancreas per la cura del diabete tipo I. È quanto asserisce il Dott. Hiro Nakuachi, capo fila del team di ricerche ge-netiche quando afferma di aver già generato un pancreas di topo nei ratti, trapiantato con successo, senza immunosoppressori, nel topo diabetico. Ma lo stesso sperimentatore asserisce che "Quando la tecnica coinvolge il binomio pecora-uomo, si incontrano grandi difficoltà". Vediamone i particolari. Gli scienziati sono riusciti ad ottenere embrioni di ovini contenenti cellule di Langherans di origine umana. Precisamente una cellula umana per ogni 10.000 cellule di pecora, che in questo caso funge da animale surrogato. Non è esattamente la prima volta che si realizza un esperimento del genere. Qualche anno fa un obiettivo si-mile era stato ottenuto nei maiali ove le cellule umane erano in un rapporto uno a 100.000. Ma questo obiettivo dove conduce? Elementare. Far crescere un embrione di animale contenente cellule umane numericamente valide e adeguatamente organizzate da costituire un organo da trapiantare nell'uomo senza problemi di rigetto. Nello studio di Stanford l'evoluzione dell'embrione chimera si è protratto per 28 giorni, ma si pensa ad un futuro in cui lo studio duri 70 giorni per ottenere numerose cellule umane, poiché se la quantità è troppo piccola non permetterebbe la formazione di un organo. In altri termini, siamo di fronte ad una realtà scientifica che da una parte ci conduce alla figura mitologica del centauro (metà uomo, metà cavallo), dall'altra allo strapotere della tecnocrazia. Eppure il centauro ha avuto una lunga storia nella quale erano sinterizzati pregi e difetti dell'uomo: dall'estrema saggezza all'incredibile crudeltà. Sullo strapotere della tecnocrazia si discute da tempo per i numerosi ambiti che coinvolge, non ultima la bioetica.

A scanso di equivoci diciamo subito che lo studio di Stanford è un passo avanti nella sconfitta del dolore dell'uomo; ne seguiamo l'evoluzione, ne indicheremo i benefici, ne sottolineeremo i limiti. Coinvolti direttamente nel campo dei trapianti, ricordiamo sommessamente i risultati di uno studio del NHS inglese che ha rivelato che nel 2016 sono morte 460 persone in lista di attesa di trapianto. ${ }^{1}$ Allora ben vengano e presto studi volti a rendere disponibili organi umani da chimere per l'uomo. D'altra parte non si può dire che nel nostro Paese non siano stati fatti dei tentativi in tale genere, per lo meno a livello legislativo. È rimasto paradigmatico se non futuristico il documento Veronesi-Dulbecco, che anticipava lo studio di Stanford, relativo al trasferimento nucleare di cellule staminali autologhe (Tnsa), denominato la via italiana alla clonazione terapeutica. ${ }^{2}$ La nuova linea di ricerca indicata dalla Commissione Dulbecco prevedeva l'inserimento di un nucleo di cellula adulta prelevata dal paziente in un ovocita senza il proprio nucleo e ciò per evitare lo sviluppo di embrione, ma non di cellule staminali da differenziare verso linee cellulari, per la formazione di tessuti volti a riparare o sostituire organi malati. Veronesi, forse con eccesso di ottimismo, affermava che "se la tecnica funzionerà - ma penso di sì - potremo ricostituire molti organi senza ricorrere al trapianto d'organo". Niente di fatto, il progetto è saltato, forse anche perché il preventivo di oltre 100 miliardi di lire è sembrato eccessivo. Eppure nel progetto c'erano degli elementi che potevano essere adeguatamente sfruttati non solo a favore dei trapianti ma anche nella comprensione dei meccanismi di tossicità dei farmaci testata sulle cellule staminali.

Ma a questo punto subentra un altro fattore, la tecnocrazia al posto della tecnica. La tecnocrazia in campo della sanità e della medicina è il dominio di un gruppo dirigente che antepone, in accordo con Ivan Cavicchi, epistemologo e storico della medicina, "alle ragioni della politica quelle considerate tecnico scientifiche e vale come sovra ordinazione di una classe di amministratori su altre

Direzione Scientifica Centro di Bioetica, Regione Umbria, Perugia, Italia

\section{Corrispondenza:}

Prof. Mario Timio, Via XX Settembre 22, 06100 Perugia, Italia.

E-mail: timma@libero.it 
classi cittadini". ${ }^{3}$ La ragione tecnocratica mira a togliere di mezzo tutto ciò che non cade nel suo modello di raziona-lità perché considerato strano, irrazionale, iposcientifico. Trasportato in sanità ciò significa che il prevalere della ragione tecnocratica (sostenuta da protocolli, standard, linee guida, algoritmi, limiti) mette all'angolo la pratica della comunità. Significa - è ancora Cavicchi a sottolinearlo - "agire per modelli e non fare per obiettivi". Il punto è proprio questo. "La tecnocrazia non ha fantasia. Essa è schiava dei suoi modelli, dei suoi procedimenti razionali. I tecnocrati sono 'macchine a norma' progettate per operare secondo le norme. Una comunità invece non può essere a norma. Essa deve avere la libertà di rappresentare le norme e di decidere come usarle o di decidere di non usarle". Rappresentare le norme significa ispirarsi a valori che diano peso non solo alle aspettative del singolo ma alle esigenze morali dell'uomo. Cioè esigenze di bioetica. Vorrei conoscere le valutazioni del Comitato di Bioetica relative allo studio effettuato a Stanford e quali siano state le aspettative e i suggerimenti dei singoli componenti sulla procedura scientifica. Sotto il profilo bioetico la tecnica di Stanford che mette a disposizione organi umani senza far ricorso a embrioni umani è in linea di massima accettabile, purché si tengano fuori dall'esperimento le cellule cerebrali e i gameti e purché non se ne faccia un mercato a disposizione di gruppi elitari.

In ogni caso l'esperimento pecora-uomo fa pendant con un altro studio di cui i mass-media hanno dato notizia quasi contemporaneamente: si tratta della clonazione di due scimmie con la tecnica della pecora Dolly. Questa sì che è satura di implicazioni bioetiche. La notizia delle scimmie clonate è stata divulgata sulla prestigiosa rivista Cell, firmata da ricercatori dell'Accademia delle Scienze di Shangai, in Cina. Gli scienziati cinesi sono riusciti a manipolare con successo dei geni attivando un processo di embriogenesi "artificiale" con lo sviluppo di embrioni da introdurre in un utero "preso in prestito". E attraverso le tradizionali fasi mitotiche che consentono a uno zigote di trasformarsi in embrione e quindi in feto è stata resa possibile la gestazione dei due macachi clonati: Zhong Zhong e Hua Hua. Nel futuro si potrebbe ripetere la clonazione umana considerando che tassonomicamente dopo la scimmia viene l'uomo. Ma ciò è proibito nell'uomo. Tuttavia ciò che è proibito nell'uomo può essere lecito nell'animale. La storia della genetica ce lo insegna. Ma la domanda che emerge è questa: a che serve clonare due scimmie se non come prova generale per clonare l'uomo? Certo, utilizzare animali in laboratorio per ucciderli con la giustificazione che sia per il bene umano è sensato per tutti tranne per gli animalisti. Ma la giustificazione diretta della clonazione umana nessuno è riuscito a trovarla, perché è una violazione antropologica troppo grande. "Mettere al mondo una replica biologica di un individuo - chiosa Francesco D'Agostino, noto bioeticista - è la più grande offesa all'individualità di un essere umano, che ha il diritto a essere considerato un individuo umano personale e non la riproduzione biologica di un altro". ${ }^{5}$

In sintesi, lo sviluppo di embrioni dalle pecore da cui è possibile creare parti del corpo umano da trapiantare in persone malate, pur nella sua spettacolarità scientifica, è eticamente accettabile purché non si utilizzino embrioni umani per trarre cellule staminali. Al contrario, la notizia della clonazione di animali per scopi ancora da definire è molto inquietante, poiché tale ricerca può essere un paravento dietro al quale si nascondono ricerche fatte direttamente sull'uomo. Perciò è più che giustificato l'allarmismo bioetico, al di là di un frettoloso trionfalismo. In ogni caso attendiamo di avere ulteriori notizie basate su evidenze scientifiche, sempre pronti ad accettare il chiaro messaggio di Giovanni Berlinguer, presidente del Comitato di Bioetica: "la pecora Dolly è stata una bufala". Oggi diremmo una fake news. Ma non avevamo messo in dubbio all'inizio dell'articolo la diffusa fake news della pecora-uomo?

\section{Bibliografia}

1. Johnston C. Shortage of organ donation led to 457 deaths last year says NHS. The Guardian, 4 settembre 2017, https://www.theguardian.com/society/2017/sep/04/silencetransplants-deadly-organs-shortage-nhs (consultato: 8 marzo 2018).

2. Documento Veronesi-Dulbecco. Relazione della commissione sull'utilizzo di cellule staminali per finalità terapeutiche. Campania: Istituto Italiano di Bioetica, 28 dicembre 2000.

3. Cavicchi I. Presentazione. Keiron 2001; 9: 9-11.

4. Zhen L, Yijian C, Yan W, et al. Cloning of macaque monkeys by somatic cell nuclear transfer. Cell 2018; 172: 881-887.

5. De Agostino F. Il prossimo sarà l'uomo. Replicarlo? Una violazione. Il Giornale, 25 Gennaio 2018, http://www. ilgiornale.it/news/politica/prossimo-sar-luomo-replicarloviolazione-1486588.html (consultato: 8 marzo 2018). 